\title{
Autocrine and paracrine unpaired signaling regulate intestinal stem cell maintenance and division
}

\author{
Dani Osman ${ }^{1, \star}$, Nicolas Buchon ${ }^{1}$, Sveta Chakrabarti ${ }^{1}$, Yu-Ting Huang ${ }^{2}$, Wan-Chi Su${ }^{2}$, Mickaël Poidevin $^{3}$, \\ Yu-Chen Tsai ${ }^{2,4, \pm}$ and Bruno Lemaitre ${ }^{1, *, \pm}$ \\ ${ }^{1}$ Global Health Institute, Station 19, EPFL, 1015 Lausanne, Switzerland \\ ${ }^{2}$ Department of Life Science, Tunghai University, Taichung-Port Road, No. 181, Sec.3, Taichung 407, Taiwan \\ ${ }^{3}$ Centre de Génétique Moléculaire (CGM), CNRS, 91198 Gif-sur-Yvette, France \\ ${ }^{4}$ Tunghai University, Life Science Research Center, Taichung, Taiwan \\ *Authors for correspondence (dani.osman@epfl.ch; bruno.lemaitre@epfl.ch) \\ FThese authors contributed equally to this work \\ Accepted 2 September 2012 \\ Journal of Cell Science 125, 5944-5949 \\ (C) 2012. Published by The Company of Biologists Ltd \\ doi: 10.1242/jcs. 113100
}

\section{Summary}

The Janus kinase (JAK) signal transducer and activator of transcription (STAT) pathway is involved in the regulation of intestinal stem cell (ISC) activity to ensure a continuous renewal of the adult Drosophila midgut. Three ligands, Unpaired 1, Unpaired 2 and Unpaired 3 (Upd1, Upd2 and Upd3, respectively) are known to activate the JAK/STAT pathway in Drosophila. Using newly generated upd mutants and cell-specific RNAi, we showed that Upd1 is required throughout the fly life to maintain basal turnover of the midgut epithelium by controlling ISC maintenance in an autocrine manner. A role of Upd2 and Upd3 in basal conditions is discernible only in old gut, where they contribute to increased ISC abnormal division. Finally, upon an acute stress such as oral bacterial infection, we showed that Upd3 is released from enterocytes and has an additive effect with $\mathrm{Upd} 2$ to promote rapid epithelial regeneration. Taken together, our results show that Upd ligands are required to maintain the midgut homeostasis under both normal and pathological states.

Key words: Intestinal stem cells, JAK/STAT, Proliferation, Upd, Infection

\section{Introduction}

Recent studies on Drosophila intestinal stem cells (ISCs) have provided new insights into the regulation of stem cell activity as well as their role in maintaining tissue homeostasis. The Drosophila midgut contains multi-potent ISCs that undergo self-renewal throughout adult life and give rise to transient progenitors, the enteroblasts, that differentiate gradually into either absorptive enterocytes (ECs) or secretory entero-endocrine cells (Micchelli and Perrimon, 2006; Ohlstein and Spradling, 2006). The turnover of differentiated cells is continuous; the entire midgut being renewed within 7-10 days (Micchelli and Perrimon, 2006). In addition to their function in basal gut maintenance, ISCs are also critical to repair intestinal damage. In Drosophila, ingestion of cytotoxic compounds or exposure to enteric pathogens cause the loss of gut cells, which promotes ISC proliferation and differentiation to repair the epithelium damage (Amcheslavsky et al., 2009; Buchon et al., 2009a; Chatterjee and Ip, 2009; Jiang et al., 2009).

The Janus kinase/signal transducer and activator of transcription (JAK/STAT) and the epidermal growth factor receptor (EGFR) pathways both regulate ISC-mediated epithelium renewal. It has been shown that gut damage induces the production of secreted ligands of Unpaired and EGF families, which activate respectively the JAK/ STAT and EGFR pathways in progenitors to promote their division and differentiation, thereby establishing homeostatic regulatory loops (Buchon et al., 2009a; Buchon et al., 2010; Cronin et al., 2009; Jiang et al., 2009; Jiang et al., 2011). While a wide array of cytokines regulate the JAK/STAT pathway in mammals, only three ligands of the Unpaired family (Upd1, Upd2 and Upd3) are known to activate
Domeless, the unique receptor of the JAK-STAT pathway in Drosophila (Agaisse et al., 2003; Harrison et al., 1998). The function of Unpaired ligands in the adult midgut has been previously investigated using either a upd3 RNAi transgene or a Drosophila line carrying updl hypomorphic alleles (Buchon et al., 2009a; Lin et al., 2010). However, no clear picture has emerged concerning their expression pattern in the midgut and their individual roles in gut homeostasis. Here, we used both RNAi approach and new Drosophila lines deleted for $u p d 2$ and/or $u p d 3$ to unravel the function of all the three Upds in the renewal of midgut epithelium.

\section{Results and Discussion}

upd genes are differentially expressed in the adult midgut Previous study has shown that oral infection with the pathogenic bacterium Erwinia carotovora carotovora 15 (Ecc15) damages the gut epithelium and induces upd3 expression in enterocytes, resulting in the proliferation of ISCs and gut repair (Buchon et al., 2009a). We extended this study by quantifying the expression levels of all upds in 5-day-old flies (referred to as unchallenged) or flies collected 4 hours and 16 hours after infection with Ecc15. Fig. 1A shows that all the upd ligands were upregulated in the midgut upon Ecc15 infection, upd 3 being the most induced. To determine the sources of Upds in the midgut, we used transcriptional reporters, in situ hybridization and in vivo RNAi approaches. Both upd1 reporters (upd1-Gal4 >UAS-GFP and upd1-lacZ) and in situ hybridization experiments showed that updl is expressed in small-nucleated cells corresponding to progenitors (ISCs and enteroblasts) (Fig. 1B and supplementary 


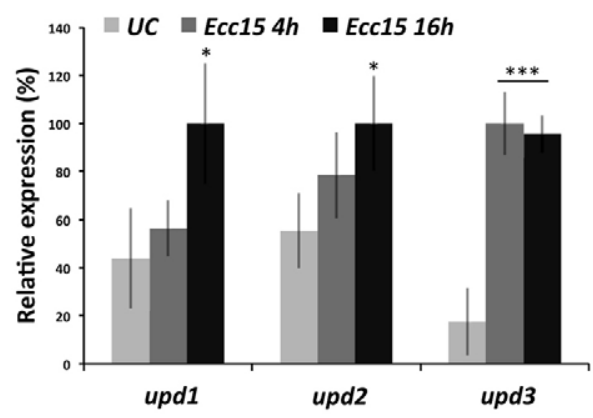

B

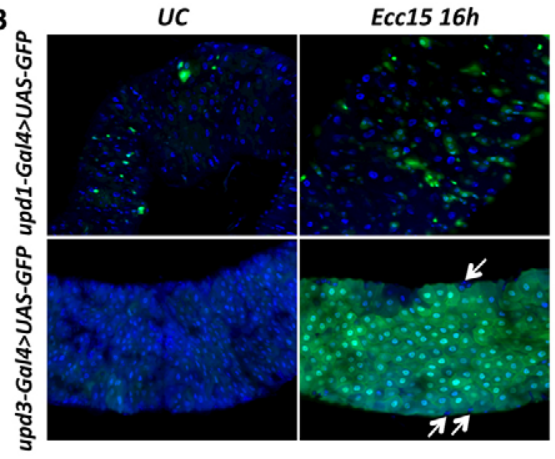

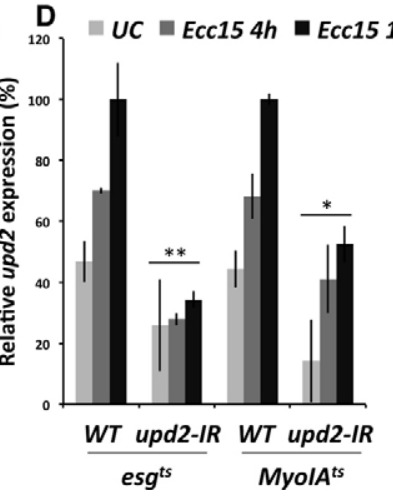

$\underset{120}{\mathrm{E}}=U C \backsim E c c 154 h \square E c c 1516 \mathrm{~h}$

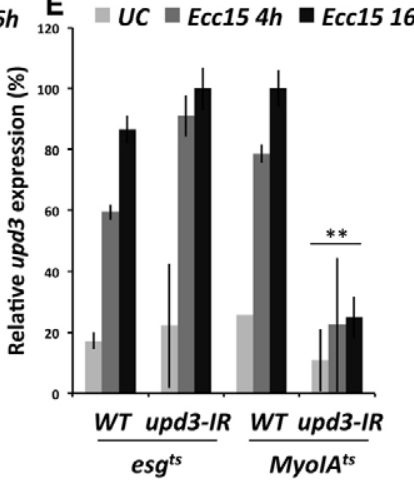

Fig. 1. upd genes are differentially expressed in the adult Drosophila midgut. (A) RT-qPCR experiments show that upd genes are induced in the gut of wild-type flies orally infected with Ecc15, compared with unchallenged (UC).

(B) Immunostaining with an anti-GFP reveals that upd1-GFP reporter is expressed in small-nucleated cells in both unchallenged and Ecc15-infected guts, whereas $u p d 3-G F P$ is strongly induced in enterocytes upon Ecc15 infection. Arrows indicate progenitor cells. (C-E) upd genes were knocked down with RNAi (IR) either in progenitors or in enterocytes of the gut using the thermosensitive Gal4 drivers Escargot-Gal4 ${ }^{t s}$ and MyoIA-Gal4 ${ }^{\text {ts }}$, respectively. upd expression was then quantified by RT-qPCR. Mean \pm s.e.m. of three experiments are shown. $* P<0.05$, ${ }^{* *} P<0.01,{ }^{* * *} P<0.001$; Student's $t$-test. material Fig. S1A,B). Following Ecc15 oral infection, the number of cells expressing upd $1>G F P$ slightly increased compared to the unchallenged condition. In contrast, upd3 $>G F P$ reporter (upd3Gal4 $>U A S-G F P)$ is weakly expressed in large nucleated enterocytes under basal condition (Fig. 1B), but it is strongly induced in most enterocytes of Ecc15 infected flies. To confirm these results, we knocked-down each Upd ligand in either progenitor cells or enterocytes using the thermosensitive Gal4 drivers EscargotGal4 $^{t s}$ and MyoIA-Gal4 ${ }^{t s}$, respectively. We then quantified by RTqPCR the expression levels of individual upd transcripts in unchallenged and Ecc15-infected guts. We observed a decrease in upd1 and upd2 expression when these genes were silenced in progenitors while both $u p d 2$ and $u p d 3$ expression was decreased when downregulated in enterocytes (Fig. 1C-E). Importantly, the knock-down of each upd in visceral muscles (How-Gal4 ${ }^{\text {ts }}>U A S$ $R N A i$ ) did not affect their expression (supplementary material Fig. $\mathrm{S} 1 \mathrm{C}$ ). Taken together, our data suggest that updl is expressed only in progenitor cells in contrast with a previous report indicating that Upd1 could be produced by the visceral muscles to regulate nonautonomously ISC proliferation (Lin et al., 2010). As upd2 expression was altered when silenced with both progenitor and enterocyte drivers, our results indicate that $u p d 2$ could be produced in both progenitors and enterocytes or in young differentiating enterocytes. Finally, our study confirmed that upd 3 expression is induced in enterocytes upon infection. To track the localization of $\mathrm{Upd} 3$, we generated a transgenic line expressing the upd3 coding sequence tagged with GFP under the control of the upd3 promoter. Although Upd3GFP was not detected in the gut of unchallenged flies, a punctate staining was observed in the basal side of the midgut epithelium after Ecc15 infection (supplementary material Fig. S2). This is consistent with the notion that $\mathrm{Upd} 3$ is released from enterocytes upon cellular damage to non-autonomously activate the ISCs scattered basally in the midgut epithelium.

\section{Upds impact JAK/STAT transcriptional activity in the midgut}

The three upd genes are clustered within a $70 \mathrm{kB}$ genomic region in the proximal part of the $X$ chromosome. It has been reported that a null mutation in updl induces embryonic lethality similar to mutations affecting the JAK kinase (hopscotch) and the STAT transcription factor (Harrison et al., 1998). To determine the function of the other Upd ligands in the midgut, we generated Drosophila lines deleted for either upd2 or upd3 that we named $u p d 2^{4}$ and $u p d 3^{4}$, respectively (supplementary material Fig. S3). Although separated by only $32 \mathrm{~kb}$, we were able to establish a $u p d 2^{4}, u p d 3^{4}$ double mutant line $\left(u p d 2,3^{\Delta}\right)$ by meiotic recombination (supplementary material Fig. S4). upd $2^{4}, u p d 3^{4}$ and $u p d 2,3^{4}$ were viable, fertile and did not show any major developmental defect, revealing that Upd1 is the only Unpaired required for viability. RTqPCR experiments on gut extracts showed that $u p d 2^{4}$ and $u p d 3^{\Delta}$ are null mutations that did not affect the expression of the other upds, indicating the absence of reciprocal transcriptional regulation (Fig. 2A,B and supplementary material Fig. S5).

We next examined the effect of each upd mutation on JAK/STAT transcriptional activity in the adult midgut. To test this, we monitored by RT-qPCR the expression of a target of JAK/STAT signaling: Socs36E (Suppressor of cytokine signaling). Fig. 2C shows that $S o c s 36 E$ expression was reduced in both $u p d 2^{4}$ and $u p d 3^{4}$ upon $E c c 15$ infection; $u p d 3^{\Delta}$ displaying a stronger effect. Importantly, no induction of $\operatorname{Socs} 36 E$ was observed in $u p d 2,3^{4}$, indicating an additive effect of $u p d 2$ and $u p d 3$ in the induction of JAK/STAT target genes upon infection (Fig. 2C). The use of the $10 \times S T A T-G F P$ transgene, a well-established reporter of JAK/STAT signaling, corroborated these results. Consistent with previous reports, STATGFP was restricted to progenitor cells in unchallenged guts, but STAT-GFP fluorescence quickly expanded in infected flies, reflecting the rapid differentiation of enteroblasts into larger 
A

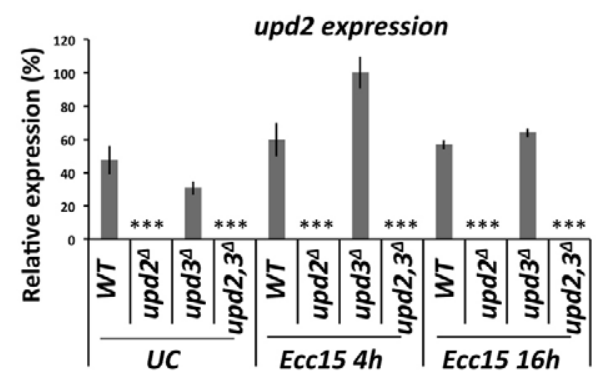

C

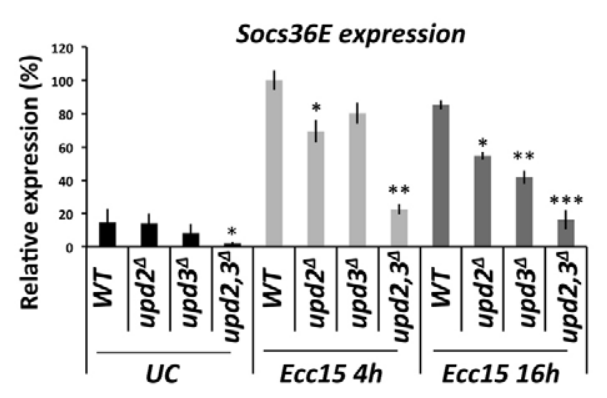

E

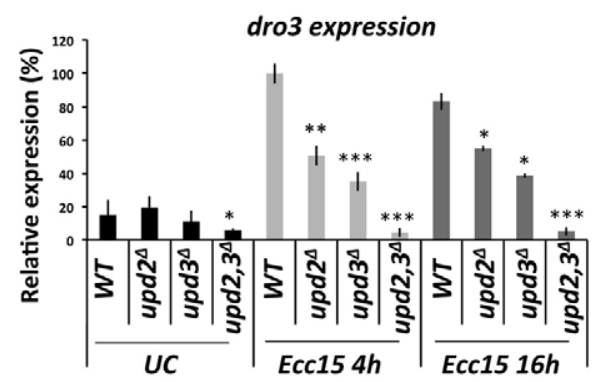

B

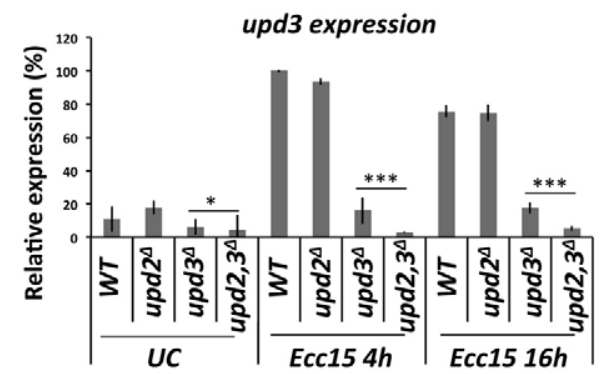

D

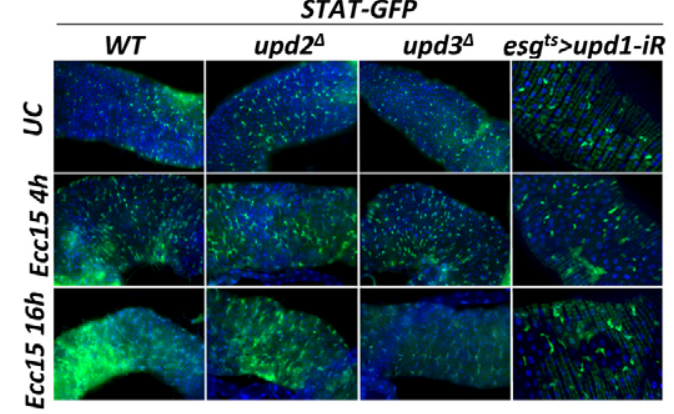

$\mathbf{F}$
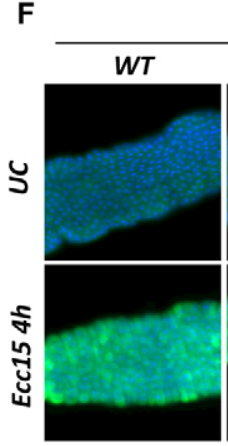

dro3-GFP upd $2^{\triangle}$

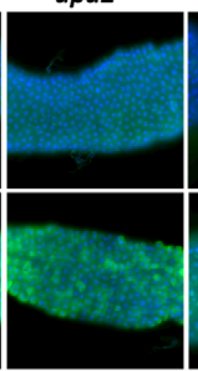

$u p d 3^{\Delta}$

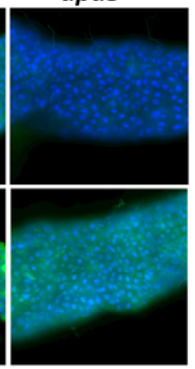

Fig. 2. Upd ligands impact JAK/STAT transcriptional activity. (A,B) RT-qPCR experiments on RNA gut extracts show that $u p d 2^{4}$ and $u p d 3^{4}$ are null mutations. Flies were either unchallenged (UC) or collected 4 hours and 16 hours after Ecc15 oral infection. (C) RT-qPCR experiments reveal that $u p d 2$ and $u p d 3$ genes regulate $\operatorname{Socs} 36 E$ expression in the gut in an additive manner. (D) $u p d 3^{4}$ or updl RNAi (esg ${ }^{t s}>$ upd1-IR) flies show a reduced expansion of $10 \times S T A T$ GFP signals in the gut upon Ecc15 infection compared to WT or upd $2^{4}$ flies.

(E) RT-qPCR experiments show that dro3 is regulated by upd 2 and $u p d 3$ in an additive manner after Ecc15 oral infection.

(F) Immunostaining with an anti-GFP shows that a $d r o 3-G F P$ reporter gene is induced in enterocytes after Ecc15 infection. dro3-GFP expression was reduced in $u p d 3^{4}$-deficient flies and to a lesser extent in upd2 flies. Data are mean \pm s.e.m. of three repeats. $* P<0.05, * * P<0.01, * * * P<0.001$; Student's $t$-test. enterocytes (Fig. 2D). In contrast to wild-type and $u p d 2^{4}$ flies, no expansion of STAT-GFP signals was observed upon Ecc15 infection in the midgut of $u p d 3^{\Delta}$ or flies with reduced updl expression (esg ${ }^{t s}$ $>$ upd1-IR, Fig. 2D).

In addition to its role in epithelium renewal, the JAK-STAT pathway contributes to antimicrobial defense in the gut since it regulates the expression of the antimicrobial peptide drosomycin3 (dro3) (Buchon et al., 2009b). RT-qPCR analysis showed that both $\mathrm{Upd} 2$ and $\mathrm{Upd} 3$ contribute to dro3 expression in the midgut of Ecc15 infected flies (Fig. 2E); Upd3 having stronger effect. To further characterize the immune role of JAK-STAT in the gut, we established a reporter transgenic fly line containing a fusion between the GFP gene and $657 \mathrm{bp}$ of the dro3 regulatory sequence upstream the start codon (dro3-GFP). Immunostaining with an anti-GFP antibody revealed almost no expression of dro3-GFP in the midgut of unchallenged flies (Fig. 2F). Interestingly, dro3-GFP was highly induced after Ecc15 infection in enterocytes of the anterior midgut (Fig. 2F, and data not shown). Consistent with the RT-qPCR results, Ecc15induced dro3-GFP expression was strongly reduced in $u p d 3^{\Delta}$ and to a lesser extent in $u p d 2^{4}$ (Fig. 2F). Altogether, we provide evidence that Upd3 is the main cytokine controlling the immune function of JAK-STAT in the gut.
Upd1 but not Upd2 or Upd3 is required for ISC maintenance To explore the contribution of each Upd in basal midgut turnover, we first used a flip-out lineage system, referred to as esg ${ }^{F / O}$ (Jiang et al., 2009). This technique enables the conditional knock down of individual upds in ISCs and their subsequent lineage, which allows tracking of both ISC proliferation and the differentiation of their newborn progeny. We found that epithelium turnover was blocked in flies with reduced expression of updl, as shown by the lack of esg ${ }^{F / O}$ clonal expansion at 8 days following the depletion (Fig. 3A). In contrast, using the esg ${ }^{F / O}$ system, the knock-down of upd2 or upd3 did not affect ISC proliferation and differentiation, as illustrated by the appearance of newly synthesized GFP-positive enterocytes along the gut (Fig. 3A). Importantly, no escargot positive cells were detectable when updl was silenced for 3 weeks in the midgut whereas inactivating upd2 or upd3 did not disrupt ISC activity (Fig. 3A). This highlights a specific role of Upd1 in the maintenance of ISC activity along the midgut in unchallenged condition. We further investigated the contribution of each Upd in basal midgut renewal by counting the number of dividing ISCs using an anti-phosphohistone H3 (anti-PH3) antibody. In agreement with the results above, updl knockdown in ISCs strongly inhibited ISC division, as evidenced by the absence of any $\mathrm{PH} 3$ positive cells compared to 

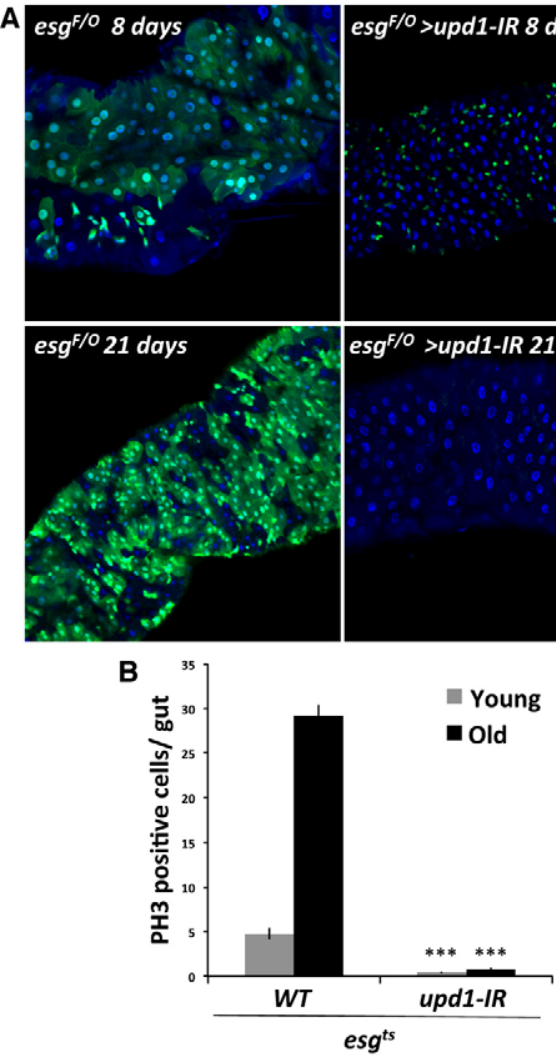
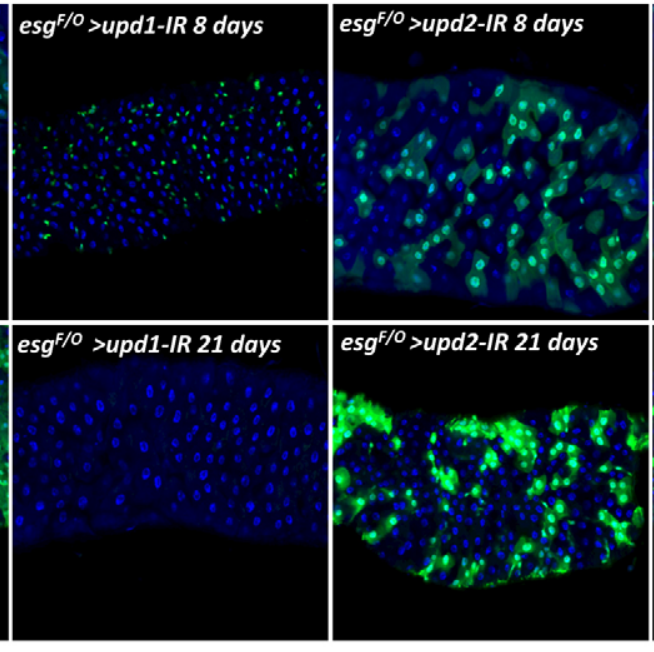

C

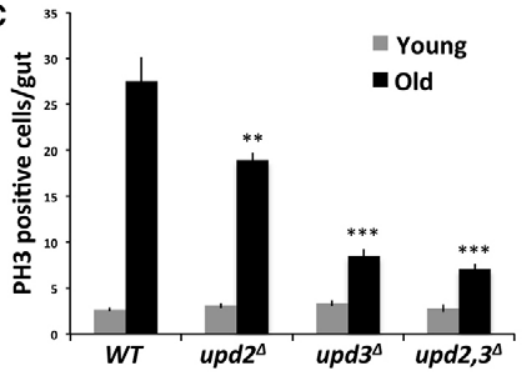

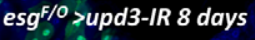
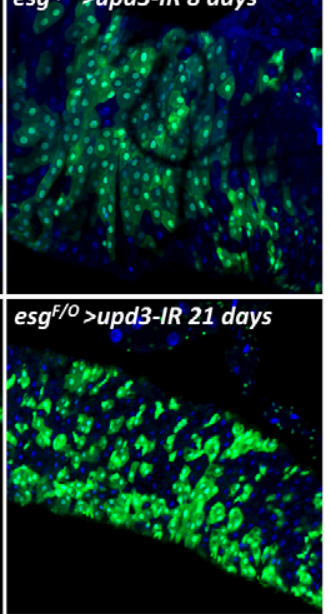

wild type (Fig. 3B). Moreover, we found a low number of PH3positive cells in midguts of upd $2^{4}, u p d 3^{4}$ and $u p d 2,3^{4}$ 5-day-old flies as in wild type (Fig. 3C).

In old flies, a deterioration of the intestinal epithelium is reflected by an aberrant enterocyte differentiation and excessive ISC proliferation. This deregulation of gut homeostasis correlated with increased signaling activities of JNK, p38b and PDGF pathways (Biteau et al., 2008; Choi et al., 2008; Park et al., 2009). We tested whether Upd ligands are part of the aberrant ISC division in aged flies. As previously reported, we observed a 10-fold higher number of $\mathrm{PH} 3$ positive cells in the midguts of 40day-old flies in comparison to 5-day-old flies (Fig. 3B,C). In contrast, the number of $\mathrm{PH} 3$ positive cells was reduced in $u p d 2^{4}$ and $u p d 3^{4}$ compared to wild-type old flies (Fig. 3C) and nearly abolished in old flies lacking updl in progenitors $\left(e s g^{t s}>\right.$ upd1$I R$, Fig. 3B). Taken together, our data highlight the requirement of Upd1 in maintaining basal gut homeostasis throughout life and reveal that Upd2 and Upd3 are involved in the increased ISC proliferation during aging.

\section{Upd2 and Upd3 regulate the gut renewal upon bacterial infection}

We finally explored the relative role of Upds in infection-induced midgut renewal. While increased ISC proliferation was observed in wild-type flies infected orally with Ecc15 (Fig. 4A), the $u p d 2^{4}$ and $u p d 3^{4}$ mutations significantly decreased the number of mitotic ISCs detected by anti-PH3 staining (Fig. 4A). Furthermore, both Upd2 and $\mathrm{Upd} 3$ contributed in an additive manner to increase the level of ISC activity as the double mutant $u p d 2,3^{4}$ displayed a lower proliferative response than each single mutant. Consistent with the role of Upd1 in ISC maintenance shown above, epithelium renewal
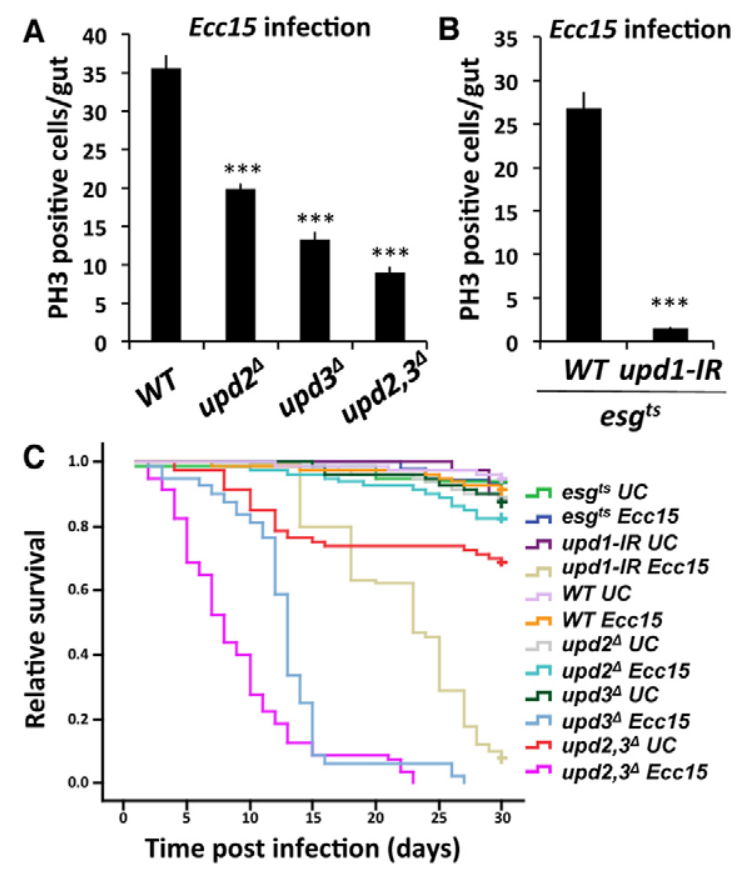

Fig. 4. The three Upds promote epithelium renewal upon Ecc15 oral infection. (A,B) Quantification of PH3-positive cells per midgut of flies dissected 8 hours after $E c c 15$ ingestion reveals that upd 2 and upd 3 promote ISC division in an additive manner (A), whereas $u p d l$ is absolutely required for ISC division (B). Mean \pm s.e.m. of three repeats are shown. ${ }^{* * *} P<0.001$; Student's $t$-test. (C) Survival analysis of females orally infected with Ecc15 at $29^{\circ} \mathrm{C}$ reveal a lower survival rate of $\operatorname{esg}^{t s}>u p d 1-I R$, upd $3^{4}$ and $u p d 2,3^{4}$ flies compared with wild type. Results are pooled data from four independent experiments, $n=20$ flies per genotype. 
was blocked in infected guts with updl knocked down (esg ${ }^{t s}$ $>$ upd1-IR, Fig. 4B). Hence, these experiments demonstrate that the three Upds participate in midgut regeneration, with a particular requirement for Upd1 in basal epithelium renewal.

The rapid regeneration of the midgut epithelium is considered as an essential component of the Drosophila defense against Ecc15 infection, as reduced JAK-STAT signaling in progenitors resulted in lower ISC proliferation and flies succumbed to Ecc15 few days following infection (Buchon et al., 2009a). To assess the role of each Upd in the resistance to Ecc15, we monitored the survival rate of flies lacking Upds after Ecc15 ingestion. Fig. 4C shows that $u p d 3^{4}$ and to a lesser extent esg $^{\text {ts }}>$ updl-IR flies succumbed within 10-15 days post-infection whereas $u p d 2^{4}$ only showed a mild susceptibility. Once more, upd $2,3^{4}$ displayed higher susceptibility than single mutant flies (Fig. 4C). These data indicate that all Upds contribute to host survival upon oral bacterial infection.

\section{Concluding remarks}

Using newly generated fly stocks deleted for upd 2 and/or upd 3 in addition to an RNAi approach, we investigated the role of three JAK/STAT ligands in the regeneration of an adult homeostatic tissue, the Drosophila midgut. Our study showed that Upd1 is produced by ISCs to control the basal activity of ISCs ensuring gut epithelium turnover. In absence of updl expression, ISCs failed to divide and were undetectable after 3 weeks, highlighting an important role of Upd1 in maintaining ISCs. In addition, we demonstrated that Upd3 is released from enterocytes, and is required to stimulate Drosophila ISC division in response to infection. Moreover, we show that $\mathrm{Upd} 2$ and $\mathrm{Upd} 3$ exhibit an additive effect in inducing ISC proliferation in infected guts. A role of Upd2 and Upd3 in absence of infection is however discernible in old flies, where they are involved in the increased ISC abnormal division probably as a response to the senescence or to the age related disruption of enterocytes. Collectively, our data draw a model in which Upd1, in agreement with its developmental function, ensure the maintenance of ISCs while $\mathrm{Upd} 2$ and Upd3 induce the replenishment of enterocytes upon damage, functioning as global stress sensors in the gut epithelium. Thus, the three ligands allow ISCs to integrate signals from different cell populations and fine-tune their activity depending on gut integrity and environment. However, we still do not fully understand how three distinct Upds can interact with the same receptor and mediate different ISC behaviors. It has been suggested that Upd1 strongly interacts with the extracellular matrix, whereas Upd2 is more diffusible (Hombría et al., 2005). Therefore, the different biophysical properties of Upds and/or their varying capacities to activate JAK/STAT signaling in terms of amplitude and duration could explain how different cytokine signaling are integrated together (Wright et al., 2011). In agreement, our findings suggest that Upd1 has a low range of action to maintain in a cell-autonomous manner basal ISC activity while Upd3 diffuses from its enterocytes source to regulate ISCs, which are scattered basally in the midgut. As different cell types express Upds, it would be interesting to unravel their differential transcriptional regulation and to better characterize the crosstalk between the Upd/JAK/STAT cascade and other signaling pathways in maintaining midgut homeostasis.

Finally, our study revealed an immune role of Upd2 and Upd3 in the midgut upon an oral bacterial infection. We showed that $\mathrm{Upd} 3$ and to a lesser extent Upd2 are involved in the transcriptional regulation of the antifungal peptide Dro3. Moreover, using a newly generated dro3-GFP reporter, we found that the regulation of $d r o 3$ by JAK-STAT is confined to the anterior compartment of the gut. How the Upd/JAK/STAT signaling pathway displays distinct immune and homeostatic activities in the adult midgut remains to be elucidated.

\section{Materials and Methods RT-qPCR}

Total RNA was extracted from 15 guts (including the crop, the cardia and the midgut) with Trizol reagent (Invitrogen) and dissolved in $10 \mathrm{uL}$ of RNase-free water. cDNA was then synthesized from 1 ug total RNA using SuperScript II enzyme (Invitrogen) and qPCR was carried out using a LightCycler 2.0 and the SYBR Green I kit (Roche). Relative expression data were normalized to RpL32.

\section{Immunostaining and in situ hybridization}

Drosophila guts were fixed for 30 minutes in PBS 0,1 Tween $4 \%$ paraformaldehyde. Blocking $(1 \mathrm{~h})$, washing $\left(10 \mathrm{~min}\right.$ each) and $4{ }^{\circ} \mathrm{C}$ overnight incubation with primary and secondary antibodies were performed in PBS 0,1 Tween 1\% BSA. Antibodies used were 1:1000 mouse anti-GFP (Roche), 1:300 mouse anti- $\beta$-galactosidase (Promega), 1:1000 rabbit anti-PH3 (Millipore), 1:500 Alexa-488 anti-mouse and 1/500 Alexa-594 anti-rabbit (Invitrogen). 1:15,000 DAPI (Sigma) was used to stain DNA. Samples were scanned with an Axioplot imager (Zeiss). Digoxigenin-labeled updl RNA probe were used for in situ hybridization as described by (Tsai and Sun, 2004). In situ hybridization experiments were performed as previously described (Osman et al., 2009 and see supplementary material Fig. S1 legend).

\section{Infection, survival experiments and fly stocks}

Erwinia carotovora carotovora 15 (Ecc15) bacterium was grown in LB medium at $29^{\circ} \mathrm{C}$ overnight. For oral infection, and after starvation $3 \mathrm{~h}$ at $29^{\circ} \mathrm{C}$, flies were flipped onto fly medium covered with filter disks soaked in a 1:1 mix of bacterial pellets at $\mathrm{OD}_{600} 200$ and $2.5 \%$ sucrose. Flies were then left to feed on this mix $4 \mathrm{~h}$ and $16 \mathrm{~h}$ for RT-qPCR or immunostaining experiments, and $24 \mathrm{~h}$ for survival analysis before being flipped onto a fresh fly medium. For fly stocks and crosses, see supplementary material Table S1.

\section{Acknowledgements}

We thank our colleague Jeremy Herren for comments on the manuscript; Harrison Douglas for updl-lacZ stock, the National Institute of Genetics (Japan); the Bloomington Stock Center; the Vienna Drosophila Resource Center; the Harvard Exelixis Stock Center and the Bruce Edgar laboratory for fly stocks. D.O., N.B. and S.C. performed the experiments. D.O., N.B., S.C. and B.L. designed the experiments and analyzed the data. D.O. and B.L. wrote the manuscript. Y.-T.H., W.-C.S. and Y.-C.T. produced upd2 and upd3 deficiencies. M.P. generated the upd3-Upd3GFP reporter.

\section{Funding}

This work was supported by a post-doctorial fellowship by the Federation of European Biochemical Societies (FEBS) (to D.O.); by the Taiwan National Science Council to Y.-C.T. [grant numbers NSC 99-2311-B-029-002-MY3]; and by the Bettencourt-Schueller Foundation; the European Research Council (ERC) Advanced Grant; and the Swiss National Fund [grant number 3100A0-12079/ 1 to B.L.].

Supplementary material available online at

http://jcs.biologists.org/lookup/suppl/doi:10.1242/jcs.113100/-/DC1

\section{References}

Agaisse, H., Petersen, U. M., Boutros, M., Mathey-Prevot, B. and Perrimon, N. (2003). Signaling role of hemocytes in Drosophila JAK/STAT-dependent response to septic injury. Dev. Cell 5, 441-450.

Amcheslavsky, A., Jiang, J. and Ip, Y. T. (2009). Tissue damage-induced intestinal stem cell division in Drosophila. Cell Stem Cell 4, 49-61.

Biteau, B., Hochmuth, C. E. and Jasper, H. (2008). JNK activity in somatic stem cells causes loss of tissue homeostasis in the aging Drosophila gut. Cell Stem Cell 3, 442-455.

Buchon, N., Broderick, N. A., Chakrabarti, S. and Lemaitre, B. (2009a). Invasive and indigenous microbiota impact intestinal stem cell activity through multiple pathways in Drosophila. Genes Dev. 23, 2333-2344. 
Buchon, N., Broderick, N. A., Poidevin, M., Pradervand, S. and Lemaitre, B. (2009b). Drosophila intestinal response to bacterial infection: activation of host defense and stem cell proliferation. Cell Host Microbe 5, 200-211.

Buchon, N., Broderick, N. A., Kuraishi, T. and Lemaitre, B. (2010). Drosophila EGFR pathway coordinates stem cell proliferation and gut remodeling following infection. BMC Biol. 8, 152.

Chatterjee, M. and Ip, Y. T. (2009). Pathogenic stimulation of intestinal stem cell response in Drosophila. J. Cell. Physiol. 220, 664-671.

Choi, N.-H., Kim, J.-G., Yang, D.-J., Kim, Y.-S. and Yoo, M.-A. (2008). Age-related changes in Drosophila midgut are associated with PVF2, a PDGF/VEGF-like growth factor. Aging Cell 7, 318-334

Cronin, S. J. F., Nehme, N. T., Limmer, S., Liegeois, S., Pospisilik, J. A., Schramek, D., Leibbrandt, A., Simoes, R. M., Gruber, S., Puc, U. et al. (2009). Genome-wide RNAi screen identifies genes involved in intestinal pathogenic bacterial infection. Science 325, 340-343.

Harrison, D. A., McCoon, P. E., Binari, R., Gilman, M. and Perrimon, N. (1998) Drosophila unpaired encodes a secreted protein that activates the JAK signaling pathway. Genes Dev. 12, 3252-3263.

Hombría, J. C.-G., Brown, S., Häder, S. and Zeidler, M. P. (2005). Characterisation of Upd2, a Drosophila JAK/STAT pathway ligand. Dev. Biol. 288, 420-433.

Jiang, H., Patel, P. H., Kohlmaier, A., Grenley, M. O., McEwen, D. G. and Edgar, B. A. (2009). Cytokine/Jak/Stat signaling mediates regeneration and homeostasis in the Drosophila midgut. Cell 137, 1343-1355.
Jiang, H., Grenley, M. O., Bravo, M.-J., Blumhagen, R. Z. and Edgar, B. A. (2011) EGFR/Ras/MAPK signaling mediates adult midgut epithelial homeostasis and regeneration in Drosophila. Cell Stem Cell 8, 84-95.

Lin, G., Xu, N. and Xi, R. (2010). Paracrine unpaired signaling through the JAK/STAT pathway controls self-renewal and lineage differentiation of drosophila intestinal stem cells. J. Mol. Cell Biol. 2, 37-49.

Micchelli, C. A. and Perrimon, N. (2006). Evidence that stem cells reside in the adult Drosophila midgut epithelium. Nature 439, 475-479.

Ohlstein, B. and Spradling, A. (2006). The adult Drosophila posterior midgut is maintained by pluripotent stem cells. Nature 439, 470-474.

Osman, D., Gobert, V., Ponthan, F., Heidenreich, O., Haenlin, M. and Waltzer, L. (2009). A Drosophila model identifies calpains as modulators of the human leukemogenic fusion protein AML1-ETO. Proc. Natl. Acad. Sci. USA 106, $12043-$ 12048.

Park, J.-S., Kim, Y.-S. and Yoo, M.-A. (2009). The role of p38b MAPK in age-related modulation of intestinal stem cell proliferation and differentiation in Drosophila. Aging (Albany NY) 1, 637-651.

Tsai, Y.-C. and Sun, Y. H. (2004). Long-range effect of upd, a ligand for Jak/STAT pathway, on cell cycle in Drosophila eye development. Genesis 39, 141-153.

Wright, V. M., Vogt, K. L., Smythe, E. and Zeidler, M. P. (2011). Differential activities of the Drosophila JAK/STAT pathway ligands Upd, Upd2 and Upd3. Cell. Signal. 23, 920-927. 